\title{
Comparison of functional outcomes after endovascular thrombectomy in patients with and without atrial fibrillation
}

\author{
James $\mathrm{Fu}^{1,2}$, Cecilia Cappelen-Smith ${ }^{1,2,3}$, Leon Edwards ${ }^{1,2,3}$, Andrew Cheung ${ }^{3,4,5}$, Nathan Manning ${ }^{4,5,6}$, Jason \\ Wenderoth $^{4,5}$, Mark Parsons ${ }^{1,2,3}$, Dennis Cordato ${ }^{1,2,3}$ \\ 'Department of Neurology and Neurophysiology, Liverpool Hospital, Sydney, NSW 2170, Australia. \\ ${ }^{2}$ South Western Sydney Clinical School, University of New South Wales, Sydney, NSW 2170, Australia. \\ ${ }^{3}$ Ingham Institute for Applied Medical Research, Sydney, NSW 2170, Australia. \\ ${ }^{4}$ Department of Interventional Neuroradiology, Liverpool Hospital, Sydney, NSW 2170, Australia. \\ ${ }^{5}$ Prince of Wales Clinical School, University of New South Wales, Sydney, NSW 2050, Australia. \\ ${ }^{6}$ The Florey Institute of Neuroscience, Melbourne, VIC 3052, Australia.
}

Correspondence to: Prof. Dennis Cordato, Department of Neurology and Neurophysiology, Liverpool Hospital, Elizabeth Street, Liverpool 2170, Sydney, NSW, Australia. E-mail: Dennis.Cordato@health.nsw.gov.au

How to cite this article: Fu J, Cappelen-Smith C, Edwards L, Cheung A, Manning N, Wenderoth J, Parsons M, Cordato D. Comparison of functional outcomes after endovascular thrombectomy in patients with and without atrial fibrillation. Vessel Plus 2021;5:33. https://dx.doi.org/10.20517/2574-1209.2021.36

Received: 24 Feb 2021 First Decision: 6 May 2021 Revised: 12 May 2021 Accepted: 27 May 2021 Published: 23 Jun 2021

Academic Editor: Aaron S. Dumont Copy Editor: Xi-Jun Chen Production Editor: Xi-Jun Chen

\begin{abstract}
Aim: Patients with atrial fibrillation (AF) are over-represented in endovascular thrombectomy (EVT) populations, due to a high prevalence of large vessel occlusions (LVO) and contraindication to intravenous thrombolysis. This study aimed to: (1) compare 90-day functional outcomes [modified Rankin Score (mRS) 0-2] and mortality in AF vs. non-AF patients receiving EVT; (2) compare 90-day functional outcomes and mortality in AF patients on therapeutic vs. non-therapeutic anticoagulation receiving EVT; and (3) identify factors influencing outcomes in AF patients receiving EVT.
\end{abstract}

Methods: A retrospective analysis of 394 consecutive patients who received EVT for anterior cerebral circulation LVO at an Australian comprehensive stroke center was performed. The main outcome measures [90-day dichotomized mRS (0-2 good; 3-6 poor functional outcome) and mortality] were compared between AF and nonAF patients, as well as between therapeutic and non-therapeutic anticoagulation cohorts. 
Results: In total, 171 (49\%) EVT patients had AF. Patients with AF were older, had higher NIHSS, and had lower rates of thrombolysis administration. AF patients showed improved 90-day mRS on multivariate analysis [aOR 1.988 (1.167-3.387)], with similar symptomatic intracranial hemorrhage ( $\mathrm{slCH})$ [aOR 0.364 (0.064-2.086)] and mortality [aOR $1.454(0.785-2.696)]$. There was no difference in 90-day mRS [aOR 1.402 (0.625-3.145)], successful reperfusion rates [aOR $3.761(0.661-21.410)$ ], or mortality [aOR $1.077(0.429-2.705)$ ] between AF patients on therapeutic vs. non-therapeutic anticoagulation. In patients with AF, advancing age and higher NIHSS were independent predictors of worse 90 -day functional outcome $(O R=1.045, P=0.020 ; O R=1.086, P=0.001)$ and mortality $(O R=1.138, P<0.001 ; O R=1.107, P=0.002)$. On multivariate analysis, thrombolysis administration improved mortality $(O R=0.215, P=0.016)$ but not functional outcomes.

Conclusion: Patients with AF showed improved 90-day functional outcome, with similar mortality and sICH, after EVT. Therapeutic anticoagulation did not adversely influence EVT outcomes.

Keywords: Endovascular thrombectomy, atrial fibrillation, anticoagulation, functional outcomes

\section{INTRODUCTION}

Endovascular thrombectomy (EVT) is the standard of care for patients with acute ischemic stroke (AIS) involving large vessel occlusion (LVO) in the anterior cerebral circulation (ACC). Five pivotal trials demonstrated that EVT improved reperfusion, neurological recovery, and functional outcomes for patients with ACC LVO, when compared to standard care alone ${ }^{[1-5]}$. Current guidelines recommend EVT after intravenous thrombolysis if eligible, for proximal ACC LVO within $6 \mathrm{~h}$ of stroke onset and within 6-24 h depending on favorable advanced imaging findings ${ }^{[6]}$.

Atrial fibrillation (AF) increases stroke risk by 4-5-fold and accounts for a third of AIS cases ${ }^{[7]}$. Patients with $\mathrm{AF}$ are also at an increased risk of a more severe AIS from a $\mathrm{LVO}^{[8]}$. The community prevalence of AF is reported as $0.51 \%{ }^{[0]}$, compared to up to $33.4 \%$ in AIS populations $s^{[7]}$ and $57.1 \%$ in LVO populations ${ }^{[10]}$. AF is commonly seen in patients undergoing EVT. Despite this, there is limited information in the literature on the impact of AF on the safety and efficacy of EVT. Previous studies have reported mixed findings, with some showing no significant difference in EVT outcomes between AF and non-AF patients ${ }^{[1,12]}$ and others reporting improved functional outcomes in $\mathrm{AF}$ patients undergoing $\mathrm{EVT}^{[13]}$.

There is even less information about the interaction between anticoagulation therapy, including warfarin and non-vitamin $\mathrm{K}$ oral anticoagulants (NOACs), and the risk of bleeding, mortality, and outcomes following EVT.

The present study was a retrospective analysis of a prospectively collected cohort of consecutive patients undergoing EVT for ACC LVO at a single comprehensive stroke center (CSC) over a four-year period. The study aimed to: (1) compare functional outcomes and mortality of AF vs. non-AF patients; (2) compare functional outcomes and mortality of AF patients on therapeutic anticoagulation $v s$. non-therapeutic anticoagulation or antithrombotic therapy; and (3) identify factors that influenced functional outcomes and mortality of AF patients undergoing EVT.

\section{METHODS}

\section{Study population}

Patients who underwent EVT for AIS between January 2016 and December 2019 were identified from a prospectively collected EVT outcomes database of Liverpool Hospital, a single CSC in Sydney, Australia. Patients with an occlusion of the ACC (internal carotid, anterior cerebral, middle cerebral M1, M2 
branches, or tandem lesions) shown by CT angiography were included. Patients with posterior circulation or distal middle cerebral artery (M3 and M4 branches) occlusions, premorbid modified Rankin Score (mRS) $\geq 3$, or those with spontaneous or post-thrombolysis reperfusion were excluded [Figure 1].

The study population was treated in accordance with current Australian guidelines, which recommends EVT for all patients with ACC occlusion within 6 or 6-24 h of stroke onset depending on favorable advanced imaging findings. All EVT cases were performed under general anesthesia, using a secondgeneration thrombectomy device, aspiration catheter, or both. No patients received intra-arterial thrombolysis.

\section{Data collection}

Detailed demographic data were collected, including age, gender, premorbid functional status defined by mRS, and stroke risk factors (atrial fibrillation, hypertension, diabetes mellitus, ischemic heart disease, congestive heart failure, current smoking, previous transient ischemic attack/stroke, and hyperlipidemia). In addition, stroke characteristics and EVT procedural data were recorded, including baseline NIHSS at time of admission, lesion location, thrombolysis administration, time to groin puncture (TTGP), and modified treatment in cerebral ischemia (mTICI) grade. Tandem lesions were defined as a severe stenosis or occlusion of the cervical carotid artery ipsilateral to the concurrent intracranial (M1/2) occlusion.

Functional outcome and mortality at 90 days, defined by mRS, was determined at the patient's three-month post-stroke follow-up appointment or by phone call for those unable to attend. The mRS score was dichotomized into "good" (mRS 0-2) and "poor" (mRS 3-6) outcomes. In AF patients, anticoagulation medication taken prior to stroke was determined through patient electronic medical records. Therapeutic anticoagulation was defined as INR $>1.9$ in those on warfarin or self-reported NOAC adherence at the time of stroke. Symptomatic intracranial hemorrhage ( $\mathrm{sICH}$ ) rates were determined according to the SITSMOST definition of $>4$ increase in NIHSS compared to baseline and parenchymal hematoma type $2^{[14]}$. Successful reperfusion was defined as mTICI $2 \mathrm{~b}$ or $2 \mathrm{c} / 3^{[15]}$.

\section{Statistical analysis}

Statistical analysis was performed using SPSS software (Version 26, IBM Corp, USA).

Baseline characteristics, including age, gender, vascular risk factors, baseline NIHSS, thrombolysis, and TTGP, were compared using descriptive statistics. Normality of continuous variables was determined by the Shapiro-Wilk test. Statistical analysis was performed using logistic regression for categorial variables, independent $t$-test for normally distributed continuous variables, and Wilcoxon rank-sum test for nonnormally distributed continuous variables.

Univariate logistic regression analysis was performed to compare dichotomized 90-day functional outcomes, mortality, reperfusion grade, and sICH between AF and non-AF cohorts, as well as AF patients on therapeutic and non-therapeutic anticoagulation. Simple logistic regression was used to identify relationships between patient and stroke characteristics, and 90-day functional outcome and mortality, in the AF cohort. Variables with $P<0.1$ significance were included in a multivariate logistic regression model to adjust for potential confounders. All results are reported as OR with $95 \%$ confidence intervals (CI). A Pvalue of $<0.05$ was considered significant.

\section{RESULTS}

In total, 464 patients underwent EVT from January 2016 to December 2019 and 349 patients were included 


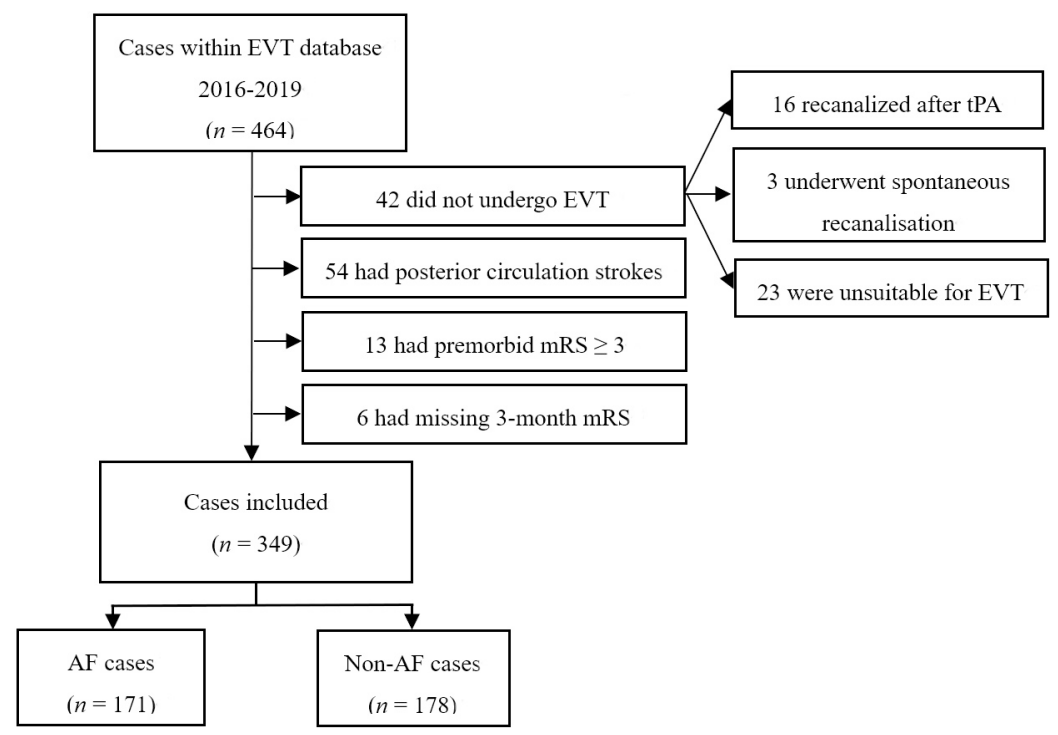

Figure 1. Inclusion and exclusion flowchart. EVT: Endovascular thrombectomy; mRS: modified Rankin Score; AF: atrial fibrillation.

in the final study, with exclusion numbers outlined in Figure 1. Of the patients included in the study, 171 (49\%) had AF and 178 (51\%) did not have AF.

\section{Baseline characteristics}

The baseline characteristics of both AF and non-AF patients are detailed in Table 1. The AF patients were significantly older $(P<0.001)$. Prevalence of IHD was higher in the AF patients $(P=0.001)$, while the prevalence of smoking was higher in the non-AF patients $(P<0.001)$.

Lesion distribution was similar, with M1 occlusions the most common. AF patients had a higher baseline NIHSS (18 vs. 17; $P=0.036$ ). The rates of thrombolysis were significantly higher in the non-AF group (44\% vs. $31 \% ; P=0.010)$. The TTGP was comparable between patient groups, with approximately two-thirds receiving EVT within $6 \mathrm{~h}$ of stroke onset. There was no difference in the number of patients who achieved successful reperfusion, but more AF patients had mTICI $2 \mathrm{c} / 3$ reperfusion.

Thirty-eight (22.2\%) of the AF patients were on therapeutic anticoagulation at the time of presentation, with $11(28.9 \%)$ on therapeutic warfarin (INR > 1.9$)$ and $27(71.1 \%)$ on NOACs. The non-therapeutic patients were older (median age $78 v$ s. $77 ; P<0.001$ ), and there was a higher proportion of females $(55.6 \% v s .36 .8 \%$; $P=0.043)$. The rate of thrombolysis was significantly higher in the non-therapeutic AF patients $(39.8 \% v s$. $0 \% ; P<0.001)$. There was no significant difference in other stroke risk factors, baseline NIHSS, lesion location, and TTGP between patient groups.

In the AF patients not on therapeutic treatment $(n=133), 70$ were on no treatment $(52.6 \%)$ at the time of stroke presentation, $42(31.6 \%)$ were taking antiplatelet therapy, and $21(15.8 \%)$ were on subtherapeutic warfarin (INR 51.9 ). Six (4.5\%) patients were non-compliant with their NOAC medication. Seventeen patients $(12.8 \%)$ had recently ceased anticoagulation, most commonly due to bleeding side effects or a planned medical procedure. Thirty-four patients (25.6\%) had newly diagnosed AF.

\section{Clinical outcomes}

There was no significant difference in rates of good functional outcome (48.0\% vs. $47.8 \%$; Figure 2$)$ on 
Table 1. Baseline characteristics of AF and non-AF patients

\begin{tabular}{|c|c|c|c|}
\hline Variables & AF & Non-AF & P-Value \\
\hline$\%$ Total & $49.0 \%(171 / 349)$ & $51.0 \%(178 / 349)$ & \\
\hline Age & & & $<0.001^{\star}$ \\
\hline Median (IQR) & $78(70-83)$ & $67(37-79)$ & \\
\hline Min-Max & $35-96$ & $29-96$ & \\
\hline Gender & & & 0.065 \\
\hline Male & $83(48.5 \%)$ & $104(58.4 \%)$ & \\
\hline Female & $88(51.5 \%)$ & $74(41.6 \%)$ & \\
\hline Hypertension & $130(76.0 \%)$ & $120(67.4 \%)$ & 0.075 \\
\hline Diabetes mellitus & $44(25.7 \%)$ & $44(24.7 \%)$ & 0.828 \\
\hline Ischemic heart disease & $65(38.0 \%)$ & $38(21.3 \%)$ & $0.001^{\star}$ \\
\hline Congestive heart failure & $19(11.1 \%)$ & $17(9.6 \%)$ & 0.632 \\
\hline Smoking & $20(11.7 \%)$ & $48(27.0 \%)$ & $<0.001^{\star}$ \\
\hline Previous TIA/Stroke & $43(25.1 \%)$ & $30(16.9 \%)$ & 0.058 \\
\hline Hyperlipidaemia & $104(60.8 \%)$ & $101(56.7 \%)$ & 0.439 \\
\hline Therapeutic anticoagulation & $38(22.2 \%)$ & $1(0.5 \%)$ & $<0.001^{\star}$ \\
\hline Baseline NIHSS & & & $0.036^{\star}$ \\
\hline Median (IQR) & $18(12-23)$ & $17(10-22)$ & \\
\hline Lesion location & & & 0.260 \\
\hline ACA & $2(1.2 \%)$ & $1(0.6 \%)$ & \\
\hline M1 & $85(49.7 \%)$ & $84(47.2 \%)$ & \\
\hline M2 & $33(19.3 \%)$ & $30(16.9 \%)$ & \\
\hline ICA & $30(17.5 \%)$ & $24(13.5 \%)$ & \\
\hline Tandem & $19(11.1 \%)$ & $35(19.7 \%)$ & \\
\hline Multiple Locations & $2(1.2 \%)$ & $4(2.2 \%)$ & \\
\hline Thrombolysis & $53(31.0 \%)$ & $79(44.4 \%)$ & $0.010^{\star}$ \\
\hline Time to groin puncture & & & 0.714 \\
\hline$\leq 6 \mathrm{~h}$ & $113(66.1 \%)$ & $113(64.2 \%)$ & \\
\hline$>6 \mathrm{~h}$ & $58(33.9 \%)$ & $63(35.8 \%)$ & \\
\hline Time to recanalization ( $\mathrm{min}$ ) & & & 0.797 \\
\hline Median (IQR) & $314(246-492)$ & $316(245-672)$ & \\
\hline mTICI Grade $2 c / 3$ & $119(69.6 \%)$ & $103(58.2 \%)$ & $0.027^{\star}$ \\
\hline
\end{tabular}

" $P<$ 0.05. AF: Atrial fibrillation; ACA: anterior cerebral circulation; ICA: internal carotid artery; IQR: inter-quartile range; IHD: ischemic heart disease; M1: middle cerebral artery segment 1; M2: middle cerebral artery segment 2; Tandem: cervical ICA and M1 or M2; mTICl: modified thrombolysis in cerebral infarction; NIHSS: National Institute of Health Stroke Score.

univariate analysis. After adjustment for age, gender, hypertension, ischemic heart disease, smoking, previous TIA/stroke, baseline NIHSS, and thrombolysis, multivariate analysis showed improved 90-day functional outcome in AF patients after EVT (aOR 1.988; 95\%CI: 1.167-3.387). There was no significant difference in 90-day mortality and sICH between groups [Table 2].

There was no significant difference in good functional outcomes ( $47.4 \% v s .48 .1 \%)$ or mortality (26.3\% vs. $20.3 \%$ ) between AF patients on therapeutic $v s$. non-therapeutic anticoagulation, even after adjustment for age, gender, and thrombolysis. The rates of successful reperfusion (97.4\% vs. 99.2\%) and sICH (2.6\%vs. $0.8 \%$ ) were comparable between these two AF subgroups [Table 3]. 
Table 2. Clinical outcomes of AF and non-AF patients

\begin{tabular}{lllllll}
\hline Clinical outcome & AF & Non-AF & OR $(\mathbf{9 5} \% \mathbf{C I})$ & $\boldsymbol{P}$ & aOR (95\% CI) & $\boldsymbol{P}$ \\
\hline mRS 0-2 & $82(48.0 \%)$ & $85(47.8 \%)$ & $1.008(0.662-1.534)$ & 0.970 & $\mathbf{1 . 9 8 8 ( 1 . 1 6 7 - 3 . 3 8 7 ) ^ { \star }}$ & $\mathbf{0 . 0 1 1}$ \\
Mortality & $37(21.6 \%)$ & $32(18.0 \%)$ & $0.794(0.468-1.346)$ & 0.391 & $1.454(0.785-2.696)$ & 0.234 \\
mTICl 2b-3 & $169(98.8 \%)$ & $171(96.1 \%)$ & $3.459(0.708-16.891)$ & 0.125 & $3.761(0.661-21.410)$ & 0.135 \\
sICH & $2(1.2 \%)$ & $7(3.9 \%)$ & $0.289(0.059-1.412)$ & 0.125 & $0.364(0.064-2.086)$ & 0.257 \\
\hline
\end{tabular}

" $P<0.05$. Adjusted odds ratio (aOR) adjusted for age, gender, hypertension, ischemic heart disease, smoking, previous TIA/stroke, baseline NIHSS, and thrombolysis. Mortality defined as $\mathrm{mRS}=6 ; \mathrm{sICH}$ defined as $>4$ increase in baseline NIHSS and parenchymal hematoma type 2. OR: Odds ratio; AF: atrial fibrillation; mRS: modified Rankin Score; $\mathrm{mTICl}$ : modified treatment in cerebral infarction score; sICH: symptomatic intracerebral hemorrhage.

Table 3. Clinical outcomes of therapeutic and non-therapeutic anticoagulation patients

\begin{tabular}{lllllll}
\hline Clinical outcome & Warfarin/NOAC & Non-therapeutic treatment & OR (95\%Cl) & $\boldsymbol{P}$ & aOR (95\%Cl) & $\boldsymbol{P}$ \\
\hline mRS 0-2 & $18(47.4 \%)$ & $64(48.1 \%)$ & $0.970(0.471-1.997)$ & 0.935 & $1.402(0.625-3.145)$ & 0.412 \\
Mortality & $10(26.3 \%)$ & $27(20.3 \%)$ & $0.713(0.309-1.646)$ & 0.428 & $1.077(0.429-2.705)$ & 0.875 \\
mTICl 2b-3 & $37(97.4 \%)$ & $132(99.2 \%)$ & $0.280(0.017-4.590)$ & 0.373 & $0.370(0.019-7.369)$ & 0.515 \\
sICH & $1(2.6 \%)$ & $1(0.8 \%)$ & $3.568(0.218-58.415)$ & 0.373 & $2.208(0.122-39.895)$ & 0.592 \\
\hline
\end{tabular}

Adjusted odds ratio (aOR) adjusted for age, gender, and thrombolysis. Mortality defined as mRS = 6; sICH defined as $>4$ increase in baseline NIHSS and parenchymal hematoma type 2. OR: Odds ratio; mRS: modified Rankin Score; mTICl: modified treatment in cerebral infarction score; sICH: symptomatic intracerebral hemorrhage.

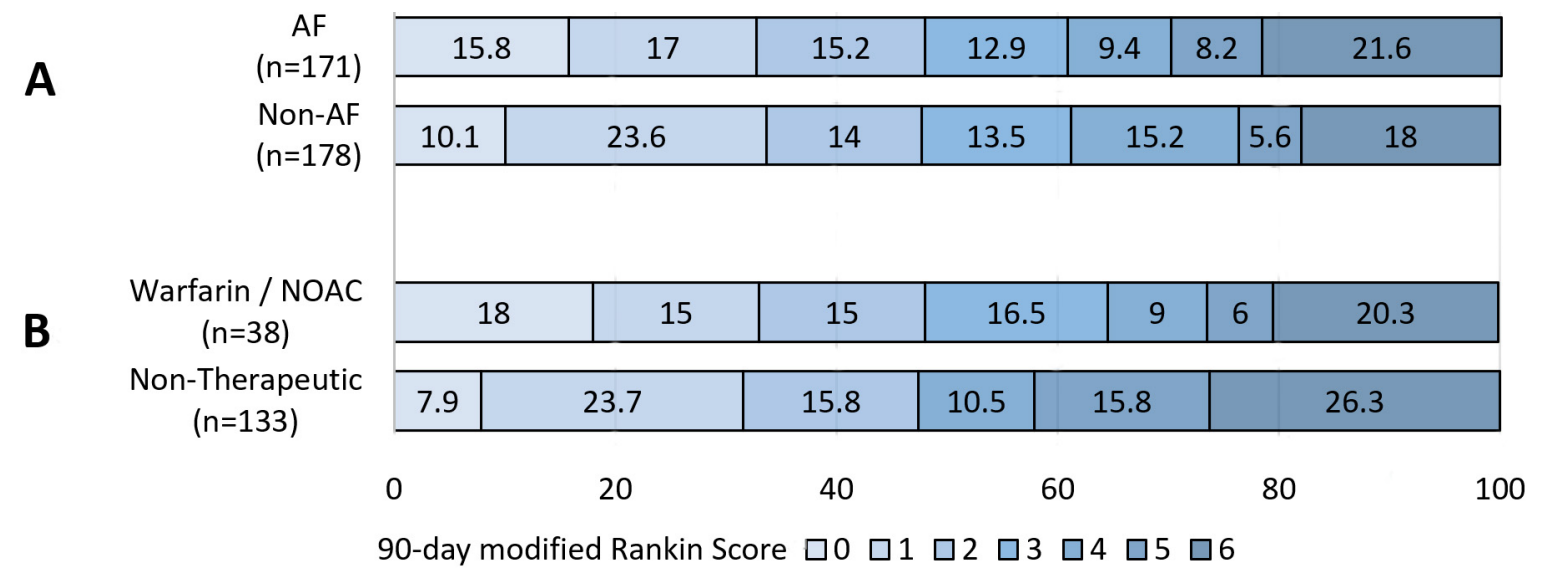

Figure 2. Distribution of 90-day modified Rankin Score by atrial fibrillation (AF) status (A) and therapeutic anticoagulation status (B) in endovascular thrombectomy patients. (B) Comparison of the AF cohort who were on therapeutic anticoagulation [warfarin/nonVitamin $\mathrm{K}$ oral anticoagulant (NOAC)] with the remainder of the cohort (subtherapeutic warfarin, non-compliant with NOAC, antiplatelet therapy, or no treatment).

\section{Factors predicting poor outcomes}

In AF patients, advancing age $(\mathrm{OR}=1.045, P=0.020$; $\mathrm{OR}=1.085, P=0.001)$ and higher baseline NIHSS $(\mathrm{OR}=1.138, P<0.001 ; \mathrm{OR}=1.107, P=0.002)$ were significant independent predictors of poor functional outcome and higher mortality at 90 days after adjustment for age, hypertension, diabetes mellitus, hyperlipidemia, baseline NIHSS, and thrombolysis [Table 4]. The administration of thrombolysis prior to EVT was independently associated with reduced mortality $(\mathrm{OR}=0.215, P=0.016)$ but not improved functional outcome $(P=0.092)$ on multivariate analysis. 
Table 4. Predictors of poor 90 -day $\mathrm{mRS}$ and higher mortality in AF group

\begin{tabular}{|c|c|c|c|c|c|c|c|c|}
\hline & \multicolumn{4}{|c|}{ Poor 90-day mRS (3-6) } & \multicolumn{4}{|c|}{ Higher mortality } \\
\hline & OR $(95 \% \mathrm{CI})$ & $\boldsymbol{P}$ & aOR' $(95 \% \mathrm{Cl})$ & $\boldsymbol{P}$ & OR $(95 \% \mathrm{Cl})$ & $\mathbf{P}$ & aOR $^{2}(95 \% \mathrm{Cl})$ & $\boldsymbol{P}$ \\
\hline Advancing age & $\begin{array}{l}1.049^{\star}(1.016- \\
1.082)\end{array}$ & $0.003^{\star}$ & $\begin{array}{l}1.045^{\star}(1.007- \\
1.085)\end{array}$ & $0.020^{\star}$ & $\begin{array}{l}1.081^{\star}(1.032- \\
1.132)\end{array}$ & $0.001^{\star}$ & $\begin{array}{l}1.085^{\star}(1.033- \\
1.142)\end{array}$ & $0.001^{\star}$ \\
\hline Male gender & $1.119(0.614-2.040)$ & 0.714 & - & - & $1.142(0.550-2.369)$ & 0.722 & - & - \\
\hline Hypertension & $2.001(0.977-4.098)$ & 0.058 & $1.625(1.681-3.879)$ & 0.274 & $\begin{array}{l}1.457(0.586- \\
3.620)\end{array}$ & 0.417 & - & - \\
\hline Diabetes mellitus & $\begin{array}{l}2.837^{\star}(1.359- \\
5.921)\end{array}$ & $0.005^{\star}$ & $\begin{array}{l}2.115(0.884- \\
5.057)\end{array}$ & 0.092 & $1.800(0.821-3.947)$ & 0.142 & - & - \\
\hline $\begin{array}{l}\text { Ischemic heart } \\
\text { disease }\end{array}$ & $1.680(0.898-3.141)$ & 0.104 & - & - & $\begin{array}{l}1.754(0.840- \\
3.660)\end{array}$ & 0.135 & - & - \\
\hline Smoking & $0.453(0.171-1.199)$ & 0.111 & - & - & $0.368(0.081-1.665)$ & 0.194 & - & - \\
\hline Previous TIA/Stroke & $1.389(0.691-2.791)$ & 0.356 & - & - & $\begin{array}{l}2.204^{\star}(1.009- \\
4.814)\end{array}$ & $0.047^{\star}$ & $\begin{array}{l}2.436(0.956- \\
6.210)\end{array}$ & 0.062 \\
\hline Hyperlipidaemia & $1.787(0.961-3.323)$ & 0.067 & $\begin{array}{l}1.349(0.626- \\
2.908)\end{array}$ & 0.445 & $\begin{array}{l}2.374^{\star}(1.040- \\
5.418)\end{array}$ & $0.040^{\star}$ & $\begin{array}{l}2.554(0.976- \\
6.684)\end{array}$ & 0.056 \\
\hline $\begin{array}{l}\text { Higher baseline } \\
\text { NIHSS }\end{array}$ & $1.141^{\star}(1.083-1.202)$ & $0.000^{\star}$ & $\begin{array}{l}1.138^{\star}(1.077- \\
1.203)\end{array}$ & $0.000^{\star}$ & $\begin{array}{l}1.100 \star(1.037- \\
1.166)\end{array}$ & $0.001^{\star}$ & $\begin{array}{l}1.107^{\star}(1.037- \\
1.183)\end{array}$ & $0.002^{\star}$ \\
\hline Thrombolysis & $\begin{array}{l}0.483^{\star}(0.249- \\
0.934)\end{array}$ & $0.031^{\star}$ & $0.515(0.238-1.115)$ & 0.092 & $\begin{array}{l}0.210^{\star}(0.070- \\
0.629)\end{array}$ & $0.005^{\star}$ & $\begin{array}{l}0.215^{\star}(0.061- \\
0.755)\end{array}$ & $0.016^{\star}$ \\
\hline Longer TTGP & $1.344(0.710-2.541)$ & 0.364 & - & & $1.928(0.917-4.056)$ & 0.084 & $\begin{array}{l}1.702(0.693- \\
4.182)\end{array}$ & 0.246 \\
\hline
\end{tabular}

'P<0.05. ${ }^{1}$ Adjusted for age, hypertension, diabetes mellitus, hyperlipidemia, baseline NIHSS, and thrombolysis administration. ${ }^{2}$ Adjusted for age, previous TIA/stroke, hyperlipidemia, baseline NIHSS, thrombolysis, and TTGP; AF group included 171 patients; poor 90-day mRS defined as mRS 3-6; mortality defined as mRS = 6. OR: Odds ratio; DM: diabetes mellitus; HT: hypertension; IHD: ischemic heart disease; NIHSS: National Institute of Health Stroke Score; TIA: transient ischemic attack.

In non-AF patients, baseline NIHSS was similarly associated with worse functional outcomes $(\mathrm{OR}=1.14, P$ $<0.001)$ and higher mortality $(\mathrm{OR}=1.085, P=0.004)$ after adjustment for the same variables, but age was only significant on univariate analysis. Thrombolysis was also associated with reduced mortality $(\mathrm{OR}=$ $0.406, P=0.039)$ in non-AF patients, but it did not improve their functional outcome $(P=0.603)$.

\section{DISCUSSION}

This study found that AF patients showed improved functional outcome at 90 days, with similar rates of mortality, successful reperfusion, and sICH. Therapeutic anticoagulation (warfarin INR > 1.9/NOACs) at the time of stroke presentation did not adversely influence EVT safety or efficacy.

In patients with AF, advancing age and higher baseline NIHSS were independent predictors of poor functional outcome and higher mortality at 90 days. Administration of thrombolysis prior to EVT was associated with significantly lower mortality on multivariate analysis and better functional outcomes in univariate analysis.

$\mathrm{AF}$ is an independent risk factor for poor outcome after AIS ${ }^{[16,17]}$. Outcome data of patients with AF who undergo EVT are less established. Our findings are supported by a recent meta-analysis, which reported higher rates of mRS 0-2 in AF patients after $\mathrm{EVT}^{[18]}$. In addition, other smaller registry studies have found similar results, suggesting improved reperfusion rates and good functional outcome in EVT patients with $\mathrm{AF}^{[13,19]}$. This may be explained by differences in underlying stroke pathophysiology. Whereas AF is typically associated with LVO of cardioembolic origin, the etiology of LVO in non-AF patients is more 
heterogeneous and can be attributed to a higher likelihood of tandem lesions or intracranial atherosclerosis, which may be associated with higher risk of complications and/or refractoriness to $\mathrm{EVT}^{[20]}$. Tandem lesions were found in $11 \%$ of our AF cohort, which makes the role of AF in the etiology of stroke uncertain and may contribute to a lower response to thrombolysis and worse 90-day outcome in this subgroup of patients ${ }^{[21]}$. Although successful reperfusion rates were similar between our AF and non-AF patients, the rate of near complete or complete reperfusion was higher in the AF cohort, which may have contributed to improved functional outcome ${ }^{[15]}$. Differences in clot characteristics and anticoagulation status among AF patients may also be associated with higher rates of first pass reperfusion and less periprocedural complications, such as distal embolism.

Other studies, including a meta-analysis of EVT patients pooling data from six landmark RCTs, found no interaction of AF on 90-day functional outcomes, mortality, or sICH rates ${ }^{[12]}$. These results have been replicated by a large registry study of United States patients undergoing EVT, which reports no difference in in-hospital mortality and hemorrhage rates in AF and non-AF patients ${ }^{[1]}$. Differences in rates of anticoagulation and thrombolysis eligibility among AF patients, rates of first pass reperfusion and patient characteristics, for example, tandem lesions or other comorbidities may explain the discrepancies found among studies. Overall, EVT has been shown to be effective and safe in LVO patients with AF.

The rate of therapeutic anticoagulation in AF patients in our study was $22.2 \%$, which is lower than a recent UK study where $41 \%$ were anticoagulated ${ }^{[22]}$. We found that therapeutic anticoagulation in AF patients at the time of stroke did not adversely influence outcome. This finding is similar to several studies, including thrombolysis-ineligible patients ${ }^{[23-27]}$.

The risk of hemorrhagic transformation is of concern in anticoagulated patients undergoing EVT. In line with our findings, other studies report no significantly increased rates of sICH in EVT patients on anticoagulation ${ }^{[2,27,28]}$. A recent study found higher rates of sICH in patients on warfarin but not on NOACs, when compared to non-anticoagulated case ${ }^{[29]}$. A previous study reported no difference in sICH rates between warfarin and NOACs, but the number of patients on a NOAC was small ${ }^{[23]}$. Further studies are required to determine the safety profiles of anticoagulation in the context of EVT.

Few studies have explored outcome predictors in AF patients undergoing EVT. Prognostic factors determining functional outcome and mortality in the literature irrespective of patient AF status include advanced age and higher baseline NIHSS ${ }^{[30-32]}$. Our study found similar outcome predictors in AF patients, suggesting that prognostic factors remain similar between AF patients and EVT patients more broadly. Increased comorbidities ${ }^{[33]}$, reduced neurological reserve ${ }^{[34]}$, and less penumbra ${ }^{[35]}$ may account for the poorer outcomes seen in older patients. All patients in our study received general anesthesia, which may have potentially influenced 90-day functional outcomes, although this is unlikely to have contributed to the significance of our AF vs. non-AF findings.

In this study, thrombolysis was identified as an independent predictor of lower mortality, although this finding was noted in both AF and non-AF patients. The benefit of thrombolysis before EVT is subject to ongoing trials (MR CLEAN NO-IV, SWIFT DIRECT, and DIRECT-SAFE) due to mixed results in observational studies ${ }^{[36-40]}$. A recent study found no additional benefit of thrombolysis, although this trial used a $20 \%$ margin of non-inferiority ${ }^{[41]}$. Current guidelines recommend thrombolysis prior to EVT in eligible patients, on the expectation that it may shorten EVT procedural time, reduce number of catheterization attempts, and dissolve residual thrombotic material ${ }^{[11,42]}$. Further studies focusing on thrombolysis in AF patients undergoing EVT may be of value. 
The limitations of our study include its retrospective design and potential for selection bias. Our data were collected from a single CSC within metropolitan Sydney, which may not be representative of other populations. Differences in baseline characteristics between AF and non-AF cohorts, including older age, higher baseline NIHSS, and lower rates of thrombolysis in those with AF, were potential confounders, which required adjustment through multivariable regression analysis. In addition, we relied on patient selfreporting for determination of NOAC use. The relatively low sample size in those on therapeutic anticoagulation did not allow us to compare warfarin and NOAC patient outcomes.

Our study found that AF patients undergoing EVT showed improved 90-day functional outcomes, with similar rates of mortality and sICH. Therapeutic anticoagulation at the time of stroke did not adversely impact on EVT outcome. AF and anticoagulation status should not influence the decision to pursue EVT. The role of bridging thrombolysis in patients with AF warrants further investigation.

\section{DECLARATIONS}

\section{Acknowledgments}

Meghan Miller for her contribution in follow-up of 3-month outcomes of patients.

\section{Authors' contributions}

Data collection: Cappelen-Smith C, Edwards L, Cheung A, Manning N, Wenderoth J, Cordato D

Data analysis: Fu J, Cappelen-Smith C, Edwards L, Parsons M, Cordato D

Study design: Cappelen-Smith C, Cordato D

Manuscript preparation: Cappelen-Smith C, Edwards L, Cheung A, Manning N, Wenderoth J, Parsons M, Cordato D

Ethics approval: Cappelen-Smith C

Interpretation: Fu J, Cappelen-Smith C, Edwards L, Cheung A, Manning N, Wenderoth J, Parsons M, Cordato D

Manuscript preparation (first and all subsequent drafts): $\mathrm{Fu} \mathrm{J}$

\section{Availability of data and materials}

Data cannot be shared due to data sharing limitations placed by the institution's approving ethics committee.

\section{Financial support and sponsorship}

None.

\section{Conflicts of interest}

All authors declare that there are no conflicts of interest.

\section{Ethical approval and consent to participate}

This study was performed in accordance with the declaration of Helsinki. Ethical approval for the study was obtained from the South Western Sydney Local Health District's Human Research Ethics Committee reference project number 2019/ETH04094. The study was a retrospective study on outcomes following endovascular thrombectomy consent was obtained for the procedure and the corresponding outcome follow up at 3 months by the operators at the time of the acute stroke.

\section{Consent for publication}

Not applicable. 


\section{Copyright}

(C) The Author(s) 2021.

\section{REFERENCES}

1. Campbell BC, Mitchell PJ, Kleinig TJ, et al; EXTEND-IA Investigators. Endovascular therapy for ischemic stroke with perfusionimaging selection. N Engl J Med 2015;372:1009-18. DOI PubMed

2. Saver JL, Goyal M, Bonafe A, et al; SWIFT PRIME Investigators. Stent-retriever thrombectomy after intravenous t-PA vs. t-PA alone in stroke. N Engl J Med 2015;372:2285-95. DOI PubMed

3. Berkhemer OA, Fransen PS, Beumer D, et al; MR CLEAN Investigators. A randomized trial of intraarterial treatment for acute ischemic stroke. N Engl J Med 2015;372:11-20. DOI PubMed

4. Goyal M, Demchuk AM, Menon BK, et al; ESCAPE Trial Investigators. Randomized assessment of rapid endovascular treatment of ischemic stroke. N Engl J Med 2015;372:1019-30. DOI PubMed

5. Jovin TG, Chamorro A, Cobo E, et al; REVASCAT Trial Investigators. Thrombectomy within 8 hours after symptom onset in ischemic stroke. N Engl J Med 2015;372:2296-306. DOI PubMed

6. Stroke Foundation. Clinical Guidelines for Stroke Management. Available from: https://informme.org.au/en/Guidelines/ClinicalGuidelines-for-Stroke-Management. [Last accessed on 10 Jun 2021].

7. Friberg L, Rosenqvist M, Lindgren A, Terént A, Norrving B, Asplund K. High prevalence of atrial fibrillation among patients with ischemic stroke. Stroke 2014;45:2599-605. DOI PubMed

8. Seachrist E, Jacob S, Ferari C, Ranasinghe T, Petrone A, Adcock A. How much does atrial fibrillation contribute to large vessel occlusions? Neurology 2019;92:P1.3-049. Available from: http://n.neurology.org/content/92/15_Supplement/P1.3.

9. Lippi G, Sanchis-Gomar F, Cervellin G. Global epidemiology of atrial fibrillation: an increasing epidemic and public health challenge. Int J Stroke 2021;16:217-21. DOI PubMed

10. Inoue M, Noda R, Yamaguchi S, et al. Specific factors to predict large-vessel occlusion in acute stroke patients. J Stroke Cerebrovasc Dis 2018;27:886-91. DOI PubMed

11. Munir MB, Alqahtani F, Beltagy A, Tarabishy A, Alkhouli M. Comparative outcomes of mechanical thrombectomy for acute ischemic stroke in patients with and without atrial fibrillation. J Vasc Interv Radiol 2017;28:1604-5. DOI PubMed

12. Smaal JA, de Ridder IR, Heshmatollah A, et al; HERMES collaboration. Effect of atrial fibrillation on endovascular thrombectomy for acute ischemic stroke. A meta-analysis of individual patient data from six randomised trials: results from the HERMES collaboration. Eur Stroke J 2020;5:245-51. DOI PubMed PMC

13. Pan X, Liu G, Li Y, et al. Is atrial fibrillation a prognostic predictor for patients with acute ischemic stroke treated with thrombectomy? Int J Clin Exp Med 2016;9:6819-24. Available from: http://www.ijcem.com/files/ijcem0020796.pdf.

14. Wahlgren N, Ahmed N, Dávalos A, et al. Thrombolysis with alteplase for acute ischaemic stroke in the Safe Implementation of Thrombolysis in Stroke-Monitoring Study (SITS-MOST): an observational study. Lancet 2007;369:275-82. DOI PubMed

15. Dargazanli C, Fahed R, Blanc R, et al; ASTER Trial Investigators. Modified thrombolysis in cerebral infarction 2C/thrombolysis in cerebral infarction 3 reperfusion should be the aim of mechanical thrombectomy: insights from the ASTER trial (contact aspiration versus stent retriever for successful revascularization). Stroke 2018;49:1189-96. DOI PubMed

16. Hannon N, Sheehan O, Kelly L, et al. Stroke associated with atrial fibrillation--incidence and early outcomes in the north Dublin population stroke study. Cerebrovasc Dis 2010;29:43-9. DOI PubMed PMC

17. Saposnik G, Gladstone D, Raptis R, Zhou L, Hart RG; Investigators of the Registry of the Canadian Stroke Network (RCSN) and the Stroke Outcomes Research Canada (SORCan) Working Group. Atrial fibrillation in ischemic stroke: predicting response to thrombolysis and clinical outcomes. Stroke 2013;44:99-104. DOI PubMed

18. Zheng J, Shi L, Xu W, et al. Impact of hyperlipidemia and atrial fibrillation on the efficacy of endovascular treatment for acute ischemic stroke: a meta-analysis. Oncotarget 2017;8:72972-84. DOI PubMed PMC

19. Lin CJ, Luo CB, Chien C, et al. Better endovascular mechanical thrombectomy outcome in atrial fibrillation patients with acute ischemic stroke: a single-center experience. J Chin Med Assoc 2020;83:756-60. DOI PubMed

20. Baek JH, Kim BM, Heo JH, Kim DJ, Nam HS, Kim YD. Outcomes of endovascular treatment for acute intracranial atherosclerosisrelated large vessel occlusion. Stroke 2018;49:2699-705. DOI PubMed

21. Nolan NM, Regenhardt RW, Koch MJ, et al. Treatment approaches and outcomes for acute anterior circulation stroke patients with tandem lesions. J Stroke Cerebrovasc Dis 2021;30:105478. DOI PubMed PMC

22. Han TS, Fry CH, Fluck D, et al. Anticoagulation therapy in patients with stroke and atrial fibrillation: a registry-based study of acute stroke care in Surrey, UK. BMJ Open 2018;8:e22558. DOI PubMed PMC

23. Zapata-Wainberg G, Ximénez-Carrillo Á, Trillo S, et al; Madrid Stroke Network. Mechanical thrombectomy in orally anticoagulated patients with acute ischemic stroke. J Neurointerv Surg 2018;10:834-8. DOI PubMed

24. Rebello LC, Haussen DC, Belagaje S, Anderson A, Frankel M, Nogueira RG. Endovascular treatment for acute ischemic stroke in the setting of anticoagulation. Stroke 2015;46:3536-9. DOI PubMed

25. Krajíčková D, Krajina A, Herzig R, et al. Safety and efficacy of mechanical thrombectomy with stent-retrievers in anticoagulated patients with anterior circulation stroke. Clin Radiol 2019;74:165.e11-6. DOI PubMed

26. Wong JWP, Churilov L, Dowling R, et al. Safety of endovascular thrombectomy for acute ischaemic stroke in anticoagulated patients ineligible for intravenous thrombolysis. Cerebrovasc Dis 2018;46:193-9. DOI PubMed

27. Benavente L, Larrosa D, García-Cabo C, et al. Safety and efficacy of mechanical thrombectomy in acute ischemic stroke of 
anticoagulated patients-a prospective observational study. J Stroke Cerebrovasc Dis 2016;25:2093-8. DOI PubMed

28. Černík D, Šaňák D, Divišová P, et al. Mechanical thrombectomy in patients with acute ischemic stroke on anticoagulation therapy. Cardiovasc Intervent Radiol 2018;41:706-11. DOI PubMed

29. Meinel TR, Kniepert JU, Seiffge DJ, et al. Endovascular stroke treatment and risk of intracranial hemorrhage in anticoagulated patients. Stroke 2020;51:892-8. DOI PubMed

30. Tajima Y, Hayasaka M, Ebihara K, Kubota M, Suda S. Predictors of poor outcome after successful mechanical thrombectomy in patients with acute anterior circulation stroke. J Clin Interv Radiol ISVIR 2017;1:139-43. DOI

31. Nogueira RG, Liebeskind DS, Sung G, Duckwiler G, Smith WS; MERCI; Multi MERCI Writing Committee. Predictors of good clinical outcomes, mortality, and successful revascularization in patients with acute ischemic stroke undergoing thrombectomy: pooled analysis of the Mechanical Embolus Removal in Cerebral Ischemia (MERCI) and Multi MERCI Trials. Stroke 2009;40:3777-83. DOI PubMed

32. Yoon W, Kim SK, Park MS, Baek BH, Lee YY. Predictive factors for good outcome and mortality after stent-retriever thrombectomy in patients with acute anterior circulation stroke. J Stroke 2017;19:97-103. DOI PubMed PMC

33. Cohen JE, Gomori JM, Leker RR. Stent retriever-based thrombectomy in octogenarians. Interv Neurol 2016;5:111-7. DOI PubMed PMC

34. Chandra RV, Leslie-Mazwi TM, Oh DC, et al. Elderly patients are at higher risk for poor outcomes after intra-arterial therapy. Stroke 2012;43:2356-61. DOI PubMed

35. To CY, Rajamand S, Mehra R, et al. Outcome of mechanical thrombectomy in the very elderly for the treatment of acute ischemic stroke: the real world experience. Acta Radiol Open 2015;4:2058460115599423. DOI PubMed PMC

36. Bracard S, Ducrocq X, Mas JL, et al. Mechanical thrombectomy after intravenous alteplase versus alteplase alone after stroke (THRACE): a randomised controlled trial. Lancet Neurology 2016;15:1138-47. DOI PubMed

37. Merlino G, Sponza M, Petralia B, et al. Short and long-term outcomes after combined intravenous thrombolysis and mechanical thrombectomy versus direct mechanical thrombectomy: a prospective single-center study. J Thromb Thrombolysis 2017;44:203-9. DOI PubMed

38. Tsivgoulis G, Katsanos AH, Mavridis D, et al. Endovascular thrombectomy with or without systemic thrombolysis? Ther Adv Neurol Disord 2017;10:151-60. DOI PubMed PMC

39. Coutinho JM, Liebeskind DS, Slater LA, et al. Combined intravenous thrombolysis and thrombectomy vs thrombectomy alone for acute ischemic stroke: a pooled analysis of the SWIFT and STAR studies. JAMA Neurol 2017;74:268-74. DOI PubMed

40. Wang H, Zi W, Hao Y, et al; ACTUAL Investigators. Direct endovascular treatment: an alternative for bridging therapy in anterior circulation large-vessel occlusion stroke. Eur J Neurol 2017;24:935-43. DOI PubMed

41. Yang P, Zhang Y, Zhang L, et al; DIRECT-MT Investigators. Endovascular thrombectomy with or without intravenous alteplase in acute stroke. N Engl J Med 2020;382:1981-93. DOI PubMed

42. Chandra RV, Leslie-Mazwi TM, Mehta BP, et al. Does the use of IV tPA in the current era of rapid and predictable recanalization by mechanical embolectomy represent good value? J Neurointerv Surg 2016;8:443-6. DOI PubMed 